Revue internationale P.M.E.

Économie et gestion de la petite et moyenne entreprise

\title{
Acquisition et conservation des ressources humaines en PME : diagnostic dans le domaine du génie-conseil
}

\section{Bruno Fabi, Denis J. Garand et Normand Pettersen}

Volume 11, numéro 2-3, 1998

URI : https://id.erudit.org/iderudit/1009043ar

DOI : https://doi.org/10.7202/1009043ar

Aller au sommaire du numéro

Éditeur(s)

Presses de l’Université du Québec

ISSN

0776-5436 (imprimé)

1918-9699 (numérique)

Découvrir la revue

Citer cet article

Fabi, B., Garand, D. J. \& Pettersen, N. (1998). Acquisition et conservation des ressources humaines en PME : diagnostic dans le domaine du génie-conseil. Revue internationale P.M.E., 11(2-3), 49-74. https://doi.org/10.7202/1009043ar
Résumé de l'article

Cet article vise à esquisser un diagnostic des pratiques d'acquisition et de conservation des ressources humaines dans 12 PME québécoises œuvrant dans le secteur du génie-conseil1. Réalisée auprès des premiers responsables en gestion des ressources humaines (GRH), cette recherche implique l'utilisation d'une grille- diagnostic exhaustive administrée par entretiens directs approfondis. Les pratiques de GRH abordées ici sont la planification des ressources humaines, l'analyse et la description des emplois, le recrutement, la sélection, l'accueil, la rémunération ainsi que l'évaluation du rendement. Les résultats visent à satisfaire trois grands objectifs de recherche. Premièrement, dresser un bilan de ces pratiques de GRH utilisées dans ces PME du génie-conseil. Deuxièmement, évaluer le niveau de développement de ces pratiques quant à /'utilisation des techniques et des connaissances disponibles en GRH. Finalement, obtenir l'appréciation des premiers responsables en GRH en ce qui concerne les difficultés éprouvées. Les résultats font ressortir le caractère adaptatif de ces PME au regard de leurs pratiques de GRH et ils mettent en évidence la pertinence d'améliorer certaines de ces pratiques.
Ce document est protégé par la loi sur le droit d'auteur. L'utilisation des services d’Érudit (y compris la reproduction) est assujettie à sa politique d'utilisation que vous pouvez consulter en ligne.

https://apropos.erudit.org/fr/usagers/politique-dutilisation/ 


\title{
Acquisition et conservation des ressources humaines en PME: diagnostic dans le domaine du génie-conseil
}

\author{
Bruno FABI \\ Université du Québec à Trois-Rivières \\ Denis J. GARAND \\ Université Laval \\ Normand PETTERSEN \\ Université du Québec à Trois-Rivières
}

\section{MOTS CLÉS}

Gestion des ressources humaines (GRH) - Pratiques de GRH Petites et moyennes entreprises (PME) - Gestion de projets (GP) Diagnostic - Sociétés-conseils - Ingénierie

\begin{abstract}
RÉSUMÉ
Cet article vise à esquisser un diagnostic des pratiques d'acquisition et de conservation des ressources humaines dans 12 PME québécoises œuvrant dans le secteur du génie-conseil'. Réalisée auprès des premiers responsables en gestion des ressources humaines (GRH), cette recherche implique l'utilisation d'une grillediagnostic exhaustive administrée par entretiens directs approfondis. Les pratiques
\end{abstract}

1. La réalisation de cette recherche a été facilitée par le soutien financier du Conseil de recherche en sciences humaines du Canada (CRSH). Nos remerciements vont également aux 12 PME qui ont gracieusement participé à l'étude.

\section{LES AUTEURS}

Bruno Fabi est professeur titulaire de gestion des ressources humaines et membre associé du GREPME. Denis J. Garand est professeur en gestion de PME et entrepreneuriat, à I'Université Laval, Québec, et collaborateur externe du GREPME. Normand Pettersen est professeur titulaire de psychologie industrielle et de méthodologie, et directeur de la maîtrise en gestion de projets. Adresse : Université du Québec à Trois-Rivières (UQTR), Département des sciences de la gestion et de l'économie, 3351, boulevard des Forges, Trois-Rivières (Québec) G9A 5H7. Téléphone: (819) 376-5080; télécopieur: (819) 376-5079. 
de GRH abordées ici sont la planification des ressources humaines, l'analyse et la description des emplois, le recrutement, la sélection, l'accueil, la rémunération ainsi que l'évaluation du rendement. Les résultats visent à satisfaire trois grands objectifs de recherche. Premièrement, dresser un bilan de ces pratiques de GRH utilisées dans ces PME du génie-conseil. Deuxièmement, évaluer le niveau de développement de ces pratiques quant à l'utilisation des techniques et des connaissances disponibles en GRH. Finalement, obtenir l'appréciation des premiers responsables en GRH en ce qui concerne les difficultés éprouvées. Les résultats font ressortir le caractère adaptatif de ces PME au regard de leurs pratiques de GRH et ils mettent en évidence la pertinence d'améliorer certaines de ces pratiques.

\section{ABSTRACT}

This paper summarises a diagnosis of some human resource management (HRM) activities in 12 small and medium-sized enterprises (SMEs) of the Canadian consulting engineering industry. Data were collected by means of thorough interviews with the persons assuming the main HRM responsibilities in these SMEs. These interviews were structured by the use of an exhaustive questionnaire covering the main HRM activities. The employment and maintenance activities analysed in this paper are human resource planning, job analysis, recruitment, selection, induction, compensation and performance appraisal. This study has three major objectives. First, it provides descriptive data on the use of these HRM activities in SMEs of the consulting engineering industry. Second, it evaluates the level of development of these activities in light of the normative HRM body of knowledge. Finally, it summarizes the major obstacles faced by the persons assuming these HRM responsibilities. The results underline the adaptive capacities of the SMEs but they also suggest the pertinence of improving some of their HRM activities.

\section{RESUMEN}

Este artículo pretende esbozar un diagnóstico de las prácticas de contratación y de conservación de los recursos humanos en 12 PyMEs de Québec, pertenecientes al sector de la consultoría técnica². Esta investigación implica la utilización de una plantilla de diagnóstico exhaustiva, elaborada a partir de entrevistas personales en profundidad, para lo que se ha contado con la participación de los máximos responsables de la gestión de recursos humanos (GRH) en las doce PyMEs. Las prácticas de recursos humanos tratadas aquí son la planificación de los recursos humanos, el análisis y la descripción de los empleos, el encuadramiento, la selección, la contratación y la remuneración, así como la evaluación del rendimiento. Los resultados pretenden satisfacer tres grandes objetivos de investigación. En primer lugar, diseñar un balance de las prácticas de GRH utilizadas en las PyMEs de consultoría técnica. En segundo lugar, evaluar el nivel de desarrollo de estas prácticas en lo que respecta a la utilización de las técnicas y de los conocimientos disponibles en GRH. Finalmente, obtener la opinión de los responsables de GRH en lo referente a las dificultades

2. La realización de esta investigación ha sido facilitada por la financiación del Conseil de recherche en sciences humaines du Canada (CRSH). Nuestro agradecimiento también a las 12 PyMEs que han participado desinteresadamente en el estudio. 
encontradas. Los resultados nos muestran el carácter adaptativo de estas PyMEs en lo que respecta a sus prácticas de GRH y ponen en evidencia la conveniencia de mejorar algunas de estas prácticas.

\section{Introduction}

Depuis deux décennies, la reconnaissance et les applications de la gestion des ressources humaines (GRH) ne cessent de s'accroître dans la majorité des organisations, apportant aux processus de gestion des préoccupations humaines autrefois plus négligées. Parmi les domaines d'activité les moins étudiés sous l'aspect des ressources humaines $(\mathrm{RH})$, on retrouve les entreprises œuvrant en gestion de projets (GP). Ces sociétés regroupent des organisations manufacturières ou industrielles (automobile, télécommunications, informatique, aérospatiale), ainsi que des entreprises de conseil et de service axées sur des projets de toutes dimensions (énergie, petits et grands ouvrages, bâtiments et travaux publics - BTP, construction, technologies de l'information). Dans la présente étude, seul le secteur du génieconseil a été retenu, notamment à cause du faible niveau de connaissances concernant les GRH dans ces firmes spécialisées. Un tel choix répond également aux multiples appels à l'utilisation d'échantillons plus homogènes en PME afin de favoriser une interprétation plus univoque des résultats obtenus (D’Amboise, 1993 ; Filion, 1990 ; Fabi et Garand, 1997 ; 1998).

Vu le peu de données empiriques sur la problématique de GRH en génieconseil, l'étude a fait appel aux récentes synthèses portant sur les pratiques de GRH en PME (Garand et Fabi, 1992, 1994 ; Fabi et Garand, 1997, 1998 ; D’Amboise et Garand, 1993, 1995) et en GP (Fabi et Pettersen, 1992 ; Harnois, 1993 ; Harnois et Fabi, 1994 ; Roux, 1994 ; Thibodeau, 1996). Afin de mieux comprendre les assises de la présente recherche, il convient ici de rappeler les grandes tendances relatives aux pratiques d'acquisition et de conservation des RH en contexte de PME.

Tout d'abord, peu d'entreprises de petite taille effectuent une réelle planification des ressources humaines (PRH) ou une véritable analyse des emplois (McEvoy, 1984) : quelques-unes établissent un plan de recrutement à court terme et mettent sur papier des descriptions de tâches, mais ces outils ne servent souvent qu'à se conformer à des normes provenant de l'extérieur (règlements, subventions, aides à l'emploi). L'absence de formalisation de cette pratique peut d'ailleurs être considérée comme une lacune majeure des PME étant donné ses répercussions sur les autres pratiques (recrutement, rémunération, formation).

Le processus de recrutement, sélection et accueil parât toutefois davantage formalisé (Benoît et Rousseau, 1993) : entre 30 et $50 \%$ des PME disposent de politiques et procédures écrites en ce domaine, parfois sous la forme d'un simple formulaire. La probabilité de mise en place augmente graduellement avec la taille, souvent sous l'unique responsabilité du propriétaire-dirigeant. 
Dans la conservation de leurs RH, les PME mettent plus d'accent sur les pratiques de rémunération et d'avantages sociaux (Hornsby et Kuratko, 1990), pour lesquelles la concurrence impose des contraintes inéluctables en offrant aux individus plus qualifiés des plans de rémunération plus attrayants. Dans un contexte économique en rapide transformation, tant à l'échelle internationale que dans la répartition des activités secondaires et tertiaires dans le tissu économique, les PME n'ont d'autre choix que de s'aligner sur les pratiques des GE.

Cependant, ces efforts ne s'étendent habituellement pas aux activités d'évaluation du rendement (appréciation du personnel). Ces pratiques demeurent nettement moins formalisées et semblent souvent appliquées en PME aux seules finalités salariales et disciplinaires, au lieu de servir des objectifs de conservation et de développement des RH (Garand et Fabi, 1994). Avec la PRH, l'analyse des emplois et l'évaluation des postes, l'évaluation du rendement constitue la pratique la moins structurée et la plus galvaudée par les entrepreneurs, lesquels effectuent bien souvent eux-mêmes une appréciation paternaliste du travail accompli par leur personnel.

Cette recension de la documentation empirique portant sur les pratiques de GRH en contexte de PME fait aussi ressortir la forte hétérogénéité des échantillons étudiés, occultant ainsi les diagnostics propres à chaque domaine d'activité. La présente recherche constitue, à notre connaissance, le premier diagnostic GRH systématique et détaillé en GP. On y poursuit trois objectifs de recherche :

1. Dresser le bilan des pratiques d'acquisition et de conservation des $\mathrm{RH}$ de ces PME.

2. Vérifier le niveau de développement (ou sophistication) de leur GRH quant à l'utilisation des techniques et connaissances disponibles à ce jour en RH.

3. Faire l'évaluation de ces pratiques auprès des premiers responsables RH de ces organisations, en identifiant les difficultés éprouvées.

\section{Méthodologie}

\section{Spécification des pratiques de GRH}

Étudier les pratiques de GRH exige de décrire précisément l'univers ou le domaine à couvrir, et de rendre explicites un certain nombre de décisions. Premièrement, la présente recherche se limite à l'étude des pratiques habituellement sous l'égide des responsables de la GRH (Tsui, 1984 ; Tsui et Gomez-Mejia, 1988) et n'englobe pas toutes les pratiques visant à optimiser la productivité des ressources humaines. Par exemple, l'application des principes de la direction de personnel, de la communication interpersonnelle ou de la résolution de conflits sont des pratiques appliquées par les cadres hiérarchiques dans leur supervision opérationnelle quotidienne et, par conséquent, ne font pas partie de l'étude. La spécification des pratiques de GRH 
retenues s'est faite à partir des plus courantes rapportées dans les ouvrages spécialisés (Belcourt et al., 1996 ; Petit et al., 1993 ; Byars et Rue, 1984 ; Cascio, 1986 ; Dolan et Schuler, 1985 ; Milkovich et Glueck, 1985 ; Peretti, 1994 ; Sekiou et al., 1992). De plus, les recommandations du Project Management Institute en cette matière ont été considérées (PMI Standards Committee, 1996). Enfin, divers articles (Dolan, Hogue et Harbottle, 1990 ; Lawrence, 1979; Mealiea et Lee, 1990 ; Murray et Dimick, 1978; Tsui, 1984 et 1987 ; Tyson et Fell, 1986) et questionnaires de recherche (Benoît et Rousseau, 1990 ; Fabi et Jacob, 1988; Saha, 1989) ont été consultés. Les pratiques retenues pour la recherche sont: 1) la planification des ressources humaines, 2) l'analyse et la description des emplois, 3) le recrutement, 4) la sélection, 5) l'accueil, 6) la rémunération et 7) l'évaluation du rendement.

Deuxièmement, la présente démarche vise à étudier les pratiques de GRH excluant le service ou l'unité administrative qui en est responsable (Ulrich, 1989). Cette décision tient du fait que plusieurs organisations, en raison de leur petite taille, n'ont pas de véritable service de GRH et qu'elles ont attribué cette responsabilité à un ou plusieurs cadres linéaires. Par contre, le profil de ces responsables GRH fera partie de l'étude.

\section{Instrument de mesure}

L'enquête a été effectuée à l'aide d'un questionnaire-diagnostic conçu pour être rempli à partir d'une entrevue semi-structurée auprès du responsable de la fonction GRH (Fabi et Pettersen, 1994). Le questionnaire-diagnostic comprend trois parties. La première partie, de loin la plus importante, porte sur les huit pratiques de GRH identifiées antérieurement. D'abord, il y a les questions qui permettent de faire le bilan proprement dit des techniques et des outils utilisés. Ce sont des questions factuelles qui invitent le répondant à décrire en détail comment les pratiques de GRH sont appliquées dans leur organisation. Ensuite, on retrouve les questions faisant appel aux opinions du répondant, qui est, rappelons-le, responsable de la GRH. Ces questions visent à connaître son appréciation à l'égard des pratiques ainsi que sa perception des éventuels besoins d'amélioration. Plus spécifiquement, les questions abordent les sujets suivants : 1) dans quelle mesure les pratiques sont satisfaisantes, 2) les principales difficultés posées, 3) les solutions à envisager, 4) l'importance qui est accordée actuellement à ces pratiques par l'organisation et, finalement, 5) l'importance qui devrait leur être accordée. Il faut noter en outre que l'étude porte essentiellement sur les pratiques de GRH relatives aux gestionnaires de projets, très majoritairement des ingénieurs de formation. Ainsi, tout au long des entretiens, les répondants ont dû limiter leurs réponses à cette seule catégorie de personnel, excluant les salariés et autres cadres de l'organisation.

Quand on se réfère à l'approche de contingence, on sait que plusieurs facteurs peuvent avoir une influence sur les pratiques de GRH (Fabi, Garand et Pettersen, 
1993). Par conséquent, les facteurs susceptibles d'être les plus pertinents ont été intégrés à la grille-diagnostic. Identifiés à la suite d'une synthèse de la documentation, notamment les travaux de Jackson, Schuler et Rivero (1989) et de Saha (1989), les facteurs retenus ont été ensuite classés dans deux autres parties du questionnaire intitulées «Profil de l'organisation » et «Profil du responsable GRH ». Les facteurs relatifs à l'organisation sont (par ordre d'apparition dans le questionnaire): 1) le type de propriété, 2) le secteur d'activités, (3) sa taille, 4) son âge, 5) sa marge de manœuvre financière, 6 ) le taux de syndicalisation et 7) son orientation stratégique. Les principaux facteurs pour le responsable GRH sont : 1) sa formation théorique 2) sa formation professionnelle et 3 ) son expérience. L'élaboration du questionnairediagnostic a nécessité plusieurs étapes : ateliers auprès d'étudiants diplômés et auprès de praticiens de la GRH ou de la GP, rencontres avec des professeurs spécialisés en gestion de projet et prétest auprès de deux responsables GRH provenant de firmes en génie-conseil (Fabi et Pettersen, 1992).

\section{Collecte des données}

La collecte des données a été effectuée au moyen d'une entrevue structurée auprès du premier responsable de la gestion des ressources humaines dans chacune des firmes. Lorsque ce poste n'existait pas officiellement, notamment dans les entreprises de petite taille, on s'adressait alors à la personne responsable de cette gestion. Muni d'un questionnaire-diagnostic exhaustif, l'enquêteur devait poser les questions, fournir toutes les clarifications nécessaires et remplir le questionnaire à partir des réponses formulées par le répondant. Ce dernier avait en main une copie du questionnaire de manière à suivre le déroulement de l'entrevue. Les entrevues avaient une durée de deux à quatre heures. Étant donné l'ampleur des informations à recueillir et le niveau de complexité de certaines questions, il était important de procéder ainsi par entrevue individuelle afin de pouvoir, d'une part, donner les explications additionnelles lorsque requises et, $d$ 'autre part, contrôler directement la qualité des informations fournies. La collecte des données s'est échelonnée sur une période de deux mois.

\section{Échantillon}

L'étude porte sur les firmes de génie-conseil œuvrant au Québec ou y ayant leur siège social. Seules les firmes comptant 50 employés et plus ont été retenues, étant donné que le niveau de formalisation des pratiques de GRH est directement relié à la taille de l'entreprise (Fabi et Garand, 1997 ; Jackson, Schuler et Rivero, 1989) et que, sous le seuil de 50 employés, les pratiques de GRH sont habituellement très peu développées (Benoît et Rousseau, 1989). À partir de répertoires gouvernementaux et de la liste des firmes d'ingénieurs publiée par le journal Les affaires (1994), 43 firmes ont été répertoriées. De ce nombre, 12 PME ont accepté de participer à l'étude. 


\section{Résultats}

La présentation des résultats est organisée en fonction des trois parties du questionnairediagnostic : le profil des organisations, celui des responsables de la GRH et, finalement, les pratiques de GRH proprement dites. En ce qui concerne la dernière partie, la plus importante, les résultats sont présentés successivement pour chacune des pratiques. Le bilan factuel des outils et des techniques en usage est d'abord présenté; il est suivi des difficultés éprouvées par les responsables GRH.

\section{Profil des organisations et des responsables de $\mathrm{RH}$}

En premier lieu, les résultats indiquent que toutes les organisations sont spécialisées en génie-conseil, de propriété indépendante $(92 \%)$ et réparties à peu près également selon les limites de taille révélées par les travaux de l'AICQ (1990) et Céré (1992). Rappelons que toutes les entreprises de génie-conseil, comme la vaste majorité des firmes de services-conseils, ne sont ni syndiquées ni régies par des conventions collectives. Cette absence de syndicalisation a un impact majeur sur la formalisation de leurs politiques et procédures de GRH, généralement non écrites ou inexistantes dans ces organisations.

En second lieu, les PME étudiées $(\mathrm{N}=12)$ existent depuis une trentaine d'années, emploient en moyenne 167 personnes, dont environ 15 employés « temporaires » (contractuels, pigistes, etc.), mais n'ont qu'une seule personne à plein temps en GRH. Toutes les PME étudiées font appel à du personnel temporaire dans une proportion moyenne de $14 \%$ laissant entrevoir la nature cyclique de ce domaine d'activité, à la merci des contrats et soumis à tous les phénomènes d'instabilité économique et politique. Malgré ces variations conjoncturelles et une croissance qui tend à diminuer depuis 15 ans, elles enregistrent des profits et poursuivent généralement une stratégie d'expansion, particulièrement dans les nouveaux services et sur les marchés internationaux.

Enfin, les interlocuteurs interviewés, généralement le plus haut responsable de GRH de chaque organisation, occupent un poste directement relié aux RH dans la moitié des cas seulement, même si $62 \%$ de leur temps est consacré aux RH. La moitié des répondants relèvent directement du p.-d. g. et leur marge d'autorité leur permet de modifier, avec la direction, les procédures et les systèmes lorsque cela s'avère nécessaire. Ils assument ces responsabilités de GRH depuis peu (en moyenne depuis cinq ans), mais sont à l'emploi de l'organisation depuis près de 10 ans. La majorité des répondants peuvent s'appuyer sur une formation universitaire de premier cycle $(67 \%)$, majoritairement en génie et en relations industrielles. La plupart d'entre eux ont approfondi leur formation en GRH par démarche personnelle (92\%) et lors de colloques et séminaires sans attestation de qualification ( $83 \%)$. 


\section{Planification des ressources humaines}

Malgré le contexte d'urgence, l'instabilité de la demande et l'absence de syndicalisation, l'acquisition des ressources humaines semble globalement reposer sur des pratiques traditionnelles de recrutement et sélection. Ainsi, la planification des RH porte généralement sur une période inférieure à six mois, sert à gérer les embauches, départs et affectations aux équipes de projets et, dans une moindre mesure, aux promotions et aux mutations (tableau $1^{3}$ ). Cette planification repose principalement sur la programmation des projets, le jugement des gestionnaires responsables, les changements dans l'organisation et l'inventaire de main-d'œuvre. Les objectifs et politiques de l'organisation de même que les analyses budgétaires s'avèrent des sources d'information moins utilisées.

\section{TABLEAU 1 \\ Principaux processus, outils et techniques de planification des ressources humaines \\ ( $\mathbf{N}=9)$}

\begin{tabular}{|c|c|c|c|c|c|c|}
\hline & \multicolumn{6}{|c|}{ Fréquence* } \\
\hline & $0 \%$ & $25 \%$ & $50 \%$ & $75 \%$ & $100 \%$ & Moyenne \\
\hline \multicolumn{7}{|l|}{ Finalités de la PRH } \\
\hline Embauches et départs & 0 & 1 & 0 & 4 & 4 & 4,2 \\
\hline Affectation aux équipes de projets & 2 & 0 & 0 & 2 & 5 & 3,9 \\
\hline Promotions et mutations & 1 & 2 & 1 & 2 & 3 & 3,4 \\
\hline \multicolumn{7}{|l|}{ Sources d'information } \\
\hline Programmation des projets & 0 & 0 & 0 & 1 & 8 & 4,9 \\
\hline Changements dans l'organisation & 0 & 1 & 1 & 4 & 3 & 4,0 \\
\hline Inventaire : main-d'œuvre actuelle & & & & & & \\
\hline et ses qualifications & 1 & 0 & 3 & 1 & 4 & 3,8 \\
\hline \multicolumn{7}{|l|}{ Objectifs et politiques } \\
\hline de l'organisation & 1 & 2 & 1 & 3 & 2 & 3,3 \\
\hline Analyse budgétaire & 2 & 1 & 1 & 2 & 3 & 3,3 \\
\hline \multicolumn{7}{|l|}{ Techniques utilisées } \\
\hline Jugement des gestionnaires responsables & s 2 & 0 & 0 & 0 & 7 & 4,1 \\
\hline
\end{tabular}

* $0 \%=$ jamais, $25 \%=$ rarement, $50 \%=$ modérément, $75 \%=$ souvent, $100 \%=$ toujours.

Toutes les autres sources d'information et techniques de PRH demeurent sous-utilisées par les trois quarts des répondants : données sur l'offre et la demande

3. Tous les tableaux de résultats présentent les données selon l'ordre décroissant de fréquence ou d'importance. Pour la majorité des sous-questions, seuls les éléments « prépondérants » (fréquence égale ou supérieure à 4 sur 5) et les «mitigés » (plus de 3 sur 5) sont reproduits aux tableaux. Les nombres fluctuants de répondants dans les divers tableaux reflètent le nombre de PME appliquant formellement les diverses pratiques de GRH. 
de main-d'œuvre, plan de remplacement, taux de roulement du personnel (turnover), ratios de productivité ou de personnel, séries chronologiques, groupement nominal, méthode Delphi et régressions statistiques.

Seulement trois répondants admettent ne pas avoir (ou très peu) de difficultés en PRH, mais huit signalent l'imprévisibilité du marché (délais, contrats aléatoires) et des besoins de main-d'œuvre (embauche en urgence). Comme pour d'autres pratiques de GRH, on signale aussi les fortes réticences des hauts dirigeants : gestion autocratique, peu d'intérêt, refus de formaliser [2] $]^{4}$.

\section{Analyse des emplois}

Comme l'illustre le tableau 2, l'analyse et la description des emplois semblent aussi moyennement répandues, la moitié des répondants (6) ayant recours à des descriptions de tâches détaillées, les autres ne s'en servant que très peu ou pas du tout. Les techniques utilisées se limitent aux méthodes les plus simples (consultation des supérieurs hiérarchiques et des dossiers administratifs). Ainsi, qu'il soit question de consultation de documents spécialisés (CCDP, DOT) ou d'experts, d'entrevue avec le titulaire du poste, d'utilisation de ses notes ou d'observation structurée, d'analyse des incidents critiques ou de questionnaires structurés (MPDQ, GOJA, PAQ ou Hay), aucune technique formalisée n'a été relevée. Face aux difficultés d'actualisation des fonctions et des responsabilités selon les projets, contrats et clients [4], le contenu des descriptions et profils d'exigences (tableau 2) demeure dans la plupart des cas au niveau du guide de base. Sans entrer dans les détails opérationnels, qui dépendent de chaque projet, ce contenu se limite aux principaux objectifs et responsabilités, à l'identification du poste hiérarchiquement supérieur et à la description sommaire des exigences (formation, connaissances et expérience). Cette particularité semble d'ailleurs constituer un prérequis de polyvalence et de flexibilité pour ces PME de services-conseils. Ainsi, on n'y précise guère les conditions de travail, les capacités physiques, les habiletés mentales ou les aptitudes requises des gestionnaires de projet. En outre, certains répondants [3] font état de l'indifférence et des réticences du personnel et de la direction face aux descriptions de tâches et à la nécessité de les formaliser davantage. L'élaboration et la révision de ces documents s'avèrent très exigeantes, considérant le manque de ressources compétentes au SRH [2]. Enfin, deux entreprises signalent d'autres problèmes de planification des activités causés par l'inexistence de descriptions de tâches; ces problèmes se manifestent notamment en formation, évaluation du rendement ou promotion de même que lors de l'ouverture de nouveaux postes non conventionnels.

4. Les résultats entre crochets indiquent le nombre de répondants ayant fait mention des propos rapportés. 
TABLEAU 2

Principaux processus, outils et techniques d'analyse et description des emplois

$(\mathbf{N}=7)$

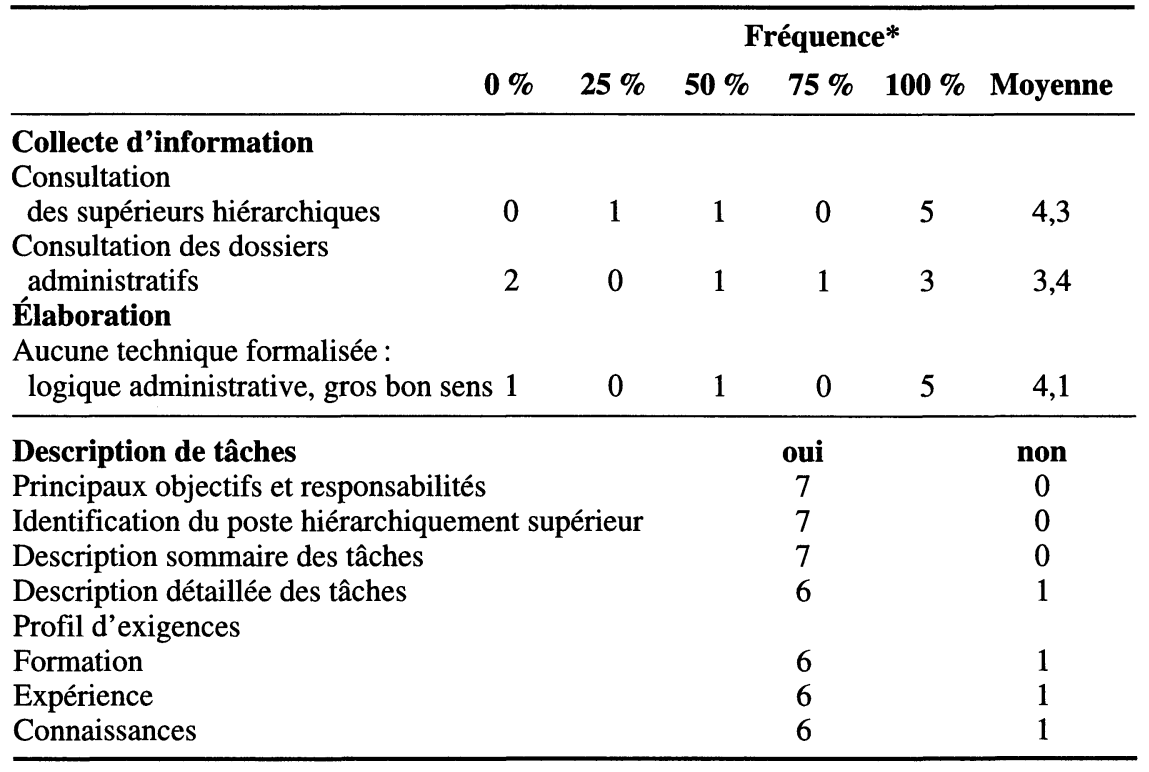

* $0 \%=$ jamais, $25 \%$ = rarement, $50 \%=$ modérément, $75 \%=$ souvent, $100 \%=$ toujours.

\section{Recrutement}

En ce qui a trait au recrutement, l'analyse du tableau 3 révèle que ces pratiques paraissent majoritairement conservatrices et peu sophistiquées quant aux techniques et outils privilégiés. La majorité des répondants [8] signalent peu de difficultés, exception faite des inconvénients relatifs à un recrutement informel, pas assez organisé, peu systématisé, décentralisé et non uniformisé [4]. En effet, les entreprises étudiées recourent à un éventail restreint de sources de recrutement et débutent par les sources les moins chères, les plus fréquemment utilisées étant les candidatures spontanées et les banques de candidats, les recommandations ou contacts personnels ainsi que les journaux locaux et nationaux. Dans cinq entreprises, on note une large utilisation des recommandations et des contacts personnels, de même que le développement plus ou moins systématisé de réseaux informels, internes et externes, chaque chef de service étant chargé de tisser sa propre toile.

Dans une moindre mesure [3], on recourt à l' affichage interne et aux revues professionnelles, tandis que les sources suivantes demeurent peu utilisées : liste de rappels, institutions d'enseignement, associations professionnelles, chasseurs de 


\section{TABLEAU 3}

\section{Principaux processus, outils et techniques de recrutement et sélection}

$$
(\mathbf{N}=\mathbf{1 2})
$$

\section{Fréquence*}

\section{$\begin{array}{lllllll}\mathbf{0} \% & \mathbf{2 5} \% & \mathbf{5 0} \% & \mathbf{7 5} \% & \mathbf{1 0 0} \% & \text { Moyenne }\end{array}$}

\section{Recrutement}

\section{Sources}

Candidatures spontanées

ou banques de candidats

Recommandations

ou contacts personnels

Journaux locaux et nationaux

Affichage interne

Revues professionnelles

$\begin{array}{llllll}0 & 1 & 3 & 4 & 4 & 3,9 \\ 1 & 0 & 3 & 4 & 4 & 3,8 \\ 1 & 2 & 4 & 2 & 3 & 3,3 \\ 5 & 2 & 1 & 2 & 2 & 2,5 \\ 4 & 3 & 5 & 0 & 0 & 2,1\end{array}$

\section{Sélection}

Techniques et outils

Analyse des curriculum vitae ou

$\begin{array}{lllllll}\text { des formulaires de demande d'emploi } & 1 & 0 & 0 & 0 & 11 & 4,7\end{array}$

Identification du niveau minimal

d'expérience

Identification du niveau minimal de scolarité

Entrevues par plus d'un intervieweur

Période de probation

$\begin{array}{llllll}2 & 0 & 0 & 1 & 9 & 4,3\end{array}$

Profil écrit de qualifications/exigences 2

Vérification des références** 0

Autres mises en situation: entrevue situationnelle

$\begin{array}{llllll}4 & 0 & 1 & 3 & 4 & 3,3\end{array}$

Guide d'entrevue écrit contenant les principales questions (entrevue semi-structurée) ${ }^{* *}$

Demande de références

$\begin{array}{lllllll}\text { (p. ex., lettre)** } & 4 & 1 & 2 & 2 & 2 & 2,7\end{array}$

Fiche de notation pour évaluer les candidats

$\begin{array}{llllll}5 & 1 & 2 & 1 & 3 & 2,7\end{array}$

$\begin{array}{lllllll}\text { Entrevues par un seul intervieweur } & 4 & 3 & 0 & 3 & 2 & 2,7\end{array}$

Description de tâches écrite

$\begin{array}{llllllll}\text { en bonne et due forme } & 5 & 2 & 1 & 1 & 3 & 2,6\end{array}$

$* 0 \%=$ jamais, $25 \%=$ rarement, $50 \%=$ modérément, $75 \%=$ souvent, $100 \%=$ toujours.

** 11 répondants.

têtes ou centres de main-d'œuvre gouvernementaux. Ainsi, quatre répondants évoquent leurs mauvaises expériences avec des agences de placement et des chasseurs de têtes, en particulier leurs coûts trop élevés et un certain piratage de main-d'œuvre. Parfois [3], on manque de temps pour organiser des concours formels et structurés, surtout pour les postes techniques. De la même façon, trois entreprises 
éprouvent des difficultés à trouver de bons candidats (ingénieurs seniors) détenant une formation très pointue, avec des connaissances et une expertise spécialisées.

L'enquête a aussi permis de constater que plusieurs PME [7] recourent à des pratiques originales de recrutement:

- recrutent de jeunes ingénieurs (MBA, M.Ing., Maîtrise en gestion de projet) par l'octroi de bourses, de stages, d'essai personnalisé, de périodes de probation, d'apprentissage ou de contrats à durée déterminée [4];

- organisent ou améliorent leur système de banque de candidats, spécialement pour les gestionnaires de projets [2];

- acceptent d'accroître la rémunération lorsque la main-d'œuvre se fait rare [1];

- collaborent régulièrement avec les DRH du secteur génie-conseil [1] ;

- adoptent des méthodes et outils sophistiqués, selon l'approche-client [1].

Par contre, les PME de génie-conseil n'effectuent, sauf exception, aucune évaluation de l'efficacité de leurs pratiques de recrutement. Seulement deux entreprises réalisent une évaluation informelle de l'efficacité de leur recrutement et prévoient éventuellement compiler des statistiques.

\section{Sélection}

Le diagnostic qui se dégage du tableau 3 indique cependant que les techniques et les outils de sélection s'avèrent plus diversifiés, tant pour l'analyse des candidatures et les entretiens de sélection que pour la validation du processus. La vaste majorité des répondants recourt à l'analyse des $\mathrm{CV}$ ou des formulaires de demande d'emploi, en identifiant un niveau minimal d'expérience et de scolarité, à l'aide d'un profil écrit de qualifications ou d'exigences et, dans une plus faible proportion, d'une description de tâches en bonne et due forme.

Les entrevues, habituellement deux par candidat, sont le plus souvent [9] effectuées par plus d'un intervieweur (en comité), ceux-ci s'appuyant davantage sur une liste de questions et diverses mises en situation informelles (entrevues situationnelles) que sur un guide d'entrevue écrit ou des instructions fournies (entretien semi-structuré). Dans trois entreprises interrogées, le premier entretien porte surtout sur les valeurs, les intérêts, la personnalité et les perceptions du candidat. Plus rarement, on ira jusqu' à une troisième ou quatrième rencontre, particulièrement pour les candidats plus importants ou appelés à l'étranger. Dans ce dernier cas, un entretien avec le conjoint ou la conjointe s'inscrit peu à peu comme une nécessité [2].

Quant aux fiches de notation, aux entrevues par un seul intervieweur et aux demandes écrites de références, elles paraissent moins utilisées pour la sélection des gestionnaires de projet. L'analyse des entretiens se déroule surtout à partir des 
notes et des commentaires personnels du comité, souvent griffonnés sur le CV. On vérifie toutefois régulièrement les références et on applique majoritairement une période de probation pour compléter le processus (tableau 3). Par ailleurs, le recours à des consultants externes, à des examens écrits, aux exercices de groupe ou à des batteries de tests fait rarement partie des outils de sélection appliqués aux gestionnaires de projets. La décision finale de sélection incombe aux supérieurs hiérarchiques, en comité, parfois avec la participation du DRH ou d'un intervenant externe comme l'Agence canadienne de développement international (ACDI) [7]. Enfin, plus de la moitié de ces PME de génie-conseil tentent d'évaluer informellement l'efficacité de leur démarche de sélection.

La majorité des répondants [8] ne rapporte aucun ou très peu de problèmes, d'erreurs commises ou d'échecs, bien qu'il y ait eu quelques rares erreurs de sélection, de promotion ou des départs impromptus. Toutefois, plusieurs [5] évoquent la formation limitée des intervieweurs : difficile évaluation des habiletés écrites, de prédiction et de validation (informelle) des tests et de la sélection, rapports d'opinions seulement, ou rigidités individuelles. Trois entreprises signalent leur difficulté à trouver des candidats ayant la culture particulière de la firme, observent des incompatibilités interpersonnelles avec le client ou des divergences internes à propos des candidats et des personnes embauchées. Trois autres répondants font état d'échecs flagrants en sélection, pour diverses raisons : aucun dossier à l'appui, besoins mal circonscrits, manque de structuration, d'uniformité, trop sur le tas, période de probation non utilisée, etc. Enfin, une seule PME a souligné le manque de temps du SRH et des membres du comité de sélection comme entrave au processus de sélection de ses gestionnaires de projets.

\section{Accueil}

Les procédures d'accueil paraissent plus développées et systématiques, l'arrivée d'un nouveau gestionnaire de projets ayant des retombées plus étendues que la venue d'un technicien ou d'un ingénieur junior. Comme l'indique le tableau 4, plusieurs informations sont presque toujours fournies lors de l'accueil, relativement aux avantages sociaux, aux services disponibles et à la philosophie de gestion, et moins fréquemment en ce qui a trait aux procédures d'assurance qualité. Plus de $80 \%$ des répondants présentent l'organisation au nouveau gestionnaire (mission, structure, technologie, etc.), offrent un tour guidé des lieux et lui expliquent ses rôles et tâches à l'aide de documents écrits et de rencontres formelles avec des collègues et des supérieurs. Cependant, ce nouvel arrivant a rarement l'occasion de faire valoir ses attentes lors de l'accueil.

Majoritairement [9], les firmes enquêtées ne font face à aucune difficulté - ou très peu - d'accueil ou d'intégration, en plus d'afficher un faible taux de roulement du personnel. Il y aurait toutefois [3] quelques lacunes de transmission de l'information 
et une communication parfois faible qui entraînent de la confusion, suscitent des rumeurs et plusieurs incertitudes. En outre, trois répondants notent que les nouveaux venus sont lancés trop rapidement dans la mêlée, sous les pressions des clients, le manque de temps et de ressources. En réalité, malgré tous les bienfaits qu'on lui accorde, le parrainage semble rarement utilisé de façon formelle dans ces PME de génie-conseil, seulement le tiers des répondants en faisant régulièrement usage. Par contre, huit des 12 firmes enquêtées déclarent appliquer une période de parrainage ou d'essai-probation, parfois informelle, allant de 3 mois (pour tous) à 6 mois pour le personnel régulier et 12 mois pour les ingénieurs seniors. Une seule entreprise pense à instaurer une évaluation après la période de probation et à officialiser alors la permanence de l'employé.

TABLEAU 4

Principaux processus, outils et techniques d'accueil

$(\mathbf{N}=\mathbf{1 2})$

\begin{tabular}{|c|c|c|c|c|c|c|}
\hline & \multicolumn{6}{|c|}{ Fréquence* } \\
\hline & $0 \%$ & $25 \%$ & $\mathbf{5 0} \%$ & $75 \%$ & $100 \%$ & Moyenne \\
\hline \multicolumn{7}{|l|}{ Informations échangées } \\
\hline $\begin{array}{l}\text { Information sur les avantages } \\
\text { sociaux }\end{array}$ & 0 & 0 & 0 & 0 & 12 & 5,0 \\
\hline $\begin{array}{l}\text { Présentation de l'organisation } \\
\text { (mission, structure, technologie, etc.) }\end{array}$ & 1 & 0 & 1 & 0 & 10 & 4,5 \\
\hline Explication des rôles et tâches & & & & & & \\
\hline du nouvel employé & 1 & 0 & 1 & 0 & 10 & 4,5 \\
\hline Information sur les services disponibles & 1 & 1 & 1 & 0 & 9 & 4,3 \\
\hline $\begin{array}{l}\text { Information sur la philosophie } \\
\text { de gestion }\end{array}$ & 1 & 0 & 3 & 2 & 6 & 4,0 \\
\hline $\begin{array}{l}\text { Information sur les procédures } \\
\text { d'assurance qualité }\end{array}$ & 1 & 3 & 3 & 1 & 4 & 3,3 \\
\hline $\begin{array}{l}\text { Présentation par le nouvel employé } \\
\text { de ses attentes }\end{array}$ & 6 & 1 & 0 & 3 & 2 & 2,5 \\
\hline Outils utilisés & & & & & & \\
\hline Visite guidée de l'organisation & 2 & 0 & 0 & 1 & 9 & 4,3 \\
\hline Documents écrits & 2 & 0 & 2 & 1 & 7 & 3,9 \\
\hline $\begin{array}{l}\text { Rencontres formelles avec collègues } \\
\text { ou supérieurs }\end{array}$ & 3 & 0 & 0 & 2 & 7 & 3,8 \\
\hline Parrainage & 8 & 0 & 0 & 1 & 3 & 2,3 \\
\hline
\end{tabular}

* $0 \%=$ jamais, $25 \%=$ rarement, $50 \%=$ modérément, $75 \%=$ souvent, $100 \%=$ toujours.

\section{Rémunération}

Les activités liées aux questions de salaires et d'avantages sociaux laissent aussi entrevoir la présence d'un certain conservatisme des pratiques de GRH appliquées dans les firmes de génie-conseil. Sauf quelques rares exceptions, les gestionnaires 
de projet reçoivent un salaire fixe $(57,5 \%)$ ou horaire $(41,7 \%)$ et un ensemble d'avantages sociaux qui s'étend parfois aux régimes d'incitation et d'intéressement, plus souvent réservés dans ces structures professionnelles aux ingénieurs associés et propriétaires. Les facteurs de détermination et de révision des salaires reposent principalement sur la capacité de payer de l'entreprise, sur les enquêtes salariales auprès des concurrents et, plus simplement, sur des ententes individuelles avec chaque gestionnaire de projet (tableau 5). Dans une moindre mesure, ces PME recourent à des niveaux établis d'exigences de l'emploi ou de qualifications, appliqués uniformément à tous les GP, et une faible minorité d'entre elles utilisent des échelles d'expérience et d'ancienneté pour la détermination et la révision des salaires.

Ainsi, comme le signale le tableau 5, les avantages sociaux offerts se limitent généralement aux programmes d'assurance collective et au paiement des frais de scolarité, la moitié des répondants n'offrant pas de régime de retraite, de prime d'éloignement ou d'autres avantages, tandis que l'autre moitié les fournit à tous ses gestionnaires. Les vacances et les congés supplémentaires (avec ou sans solde), les prêts d'argent et autres bénéfices sont rarement disponibles pour les gestionnaires de projet de ces PME. Cependant, neuf des 12 firmes étudiées offrent à leurs gestionnaires de projets l'une ou l'autre des formes suivantes d'incitation et d'intéressement :

- sept donnent accès à l'actionnariat : deux firmes l'offrent à $100 \%$ des gestionnaires de projets, deux autres à $75 \%$ et trois à $25 \%$ d'entre eux ;

- trois entreprises partagent leurs profits avec tous leurs gestionnaires de projets, et trois autres avec le quart seulement ;

- cinq répondants déclarent offrir des primes au rendement, mais une seule le fait pour tous ses gestionnaires de projets, les quatre autres le faisant pour $25 \%$;

- une seule firme partage ses gains de productivité avec le quart de ses gestionnaires de projets.

Comme les firmes de génie-conseil ne sont pas syndiquées et agissent continuellement en contexte de gestion de projets, l'éventail des régimes et des programmes de rémunération correspond davantage à un système individualisé qu'à un programme de type collectif répondant à des groupes de salariés hétérogènes et hiérarchisés [3]. Ainsi, la majorité des firmes étudiées [8] ne rapporte aucune difficulté ou insatisfaction, ou très peu, en matière de rémunération et d'avantages sociaux, même lorsqu'on doit appliquer des gels salariaux. Par contre, six répondants (dont deux qui ne révèlent aucun problème) déplorent l'absence totale de structure de rémunération et conviennent de la nécessité d'améliorer leur professionnalisme. Ces derniers ne disposent pas d'échelles salariales et œuvrent dans une entreprise privée 
TABLEAU 5

Principaux processus, outils et techniques de rémunération : salaires et avantages sociaux

$(\mathbf{N}=12)$

\begin{tabular}{|c|c|c|c|c|c|c|}
\hline & \multicolumn{6}{|c|}{ Fréquence* } \\
\hline & $0 \%$ & $25 \%$ & $50 \%$ & $75 \%$ & $100 \%$ & Moyenne \\
\hline \multicolumn{7}{|l|}{$\begin{array}{l}\text { Facteurs de détermination } \\
\text { et de révision }\end{array}$} \\
\hline Capacité de payer de l'entreprise & 1 & 0 & 3 & 1 & 7 & 4,1 \\
\hline $\begin{array}{l}\text { Etudes salariales auprès des firmes } \\
\text { du même secteur }\end{array}$ & 0 & 1 & 3 & 3 & 5 & 4,0 \\
\hline Entente individuelle & & & & & & \\
\hline $\begin{array}{l}\text { avec chacun des GP } \\
\text { Niveau d'exigence de l'emploi }\end{array}$ & $\begin{array}{l}1 \\
1\end{array}$ & $\begin{array}{l}2 \\
1\end{array}$ & $\begin{array}{l}1 \\
6\end{array}$ & $\begin{array}{l}3 \\
0\end{array}$ & $\begin{array}{l}5 \\
4\end{array}$ & $\begin{array}{l}3,8 \\
3,4\end{array}$ \\
\hline $\begin{array}{l}\text { Niveau de qualification appliqué } \\
\text { uniformément aux GP } \\
\text { Échelles d'expérience et d'ancienneté } \\
\text { appliquées uniformément }\end{array}$ & é & 1 & 4 & 2 & 3 & 3,3 \\
\hline à tous les GP & 6 & 2 & 1 & 0 & 3 & 2,3 \\
\hline \multicolumn{7}{|l|}{ Avantages sociaux offerts** } \\
\hline Régimes d'assurance collective & 0 & 0 & 1 & 0 & 11 & 4,8 \\
\hline Frais de scolarité & 1 & 2 & 1 & 0 & 8 & 4,0 \\
\hline Régimes de retraite & 5 & 0 & 1 & 0 & 6 & 3,2 \\
\hline Primes d'éloignement & 5 & 1 & 0 & 0 & 6 & 3,1 \\
\hline $\begin{array}{l}\text { Vacances et congés supplémentaires } \\
\text { sans solde }\end{array}$ & 4 & 4 & 1 & 0 & 3 & 2,5 \\
\hline \multicolumn{7}{|l|}{$\begin{array}{l}\text { Régimes d'incitation } \\
\text { et d'intéressement** }\end{array}$} \\
\hline Actionnariat & 5 & 3 & 0 & 2 & 2 & 2,4 \\
\hline Partage des profits & 7 & 3 & 0 & 0 & 2 & 1,9 \\
\hline
\end{tabular}

* $0 \%=$ jamais, $25 \%=$ rarement, $50 \%=$ modérément, $75 \%=$ souvent, $100 \%=$ toujours.

$* * 0 \%=$ pour aucun $\ldots$ à $\ldots 100 \%=$ pour tous.

peu formalisée où tout se règle au cas par cas, sans trop de discussion. Un processus d'évaluation formelle des postes leur paraît plus approprié que de se limiter au seul marché ou uniquement au système par rang. Ils éprouvent des problèmes de cohérence, d'uniformité et de transparence (équité salariale à perfectionner), mais sont freinés par le coût élevé des systèmes par points. Cette limite des capacités financières de l'organisation touche plus particulièrement quatre PME dont les budgets sont soumis aux aléas de chaque contrat et de chaque client ; il leur semble difficile d'imputer à des employés soumis à une forte imposition les coûts des avantages sociaux (p. ex., les franchises trop élevées de l'assurance collective). Deux de ces répondants admettent avoir des difficultés à répondre aux attentes des employés par l'incitation, l'intéressement ou la participation ; ils doivent également surmonter les réticences et la résistance des dirigeants face aux questions de rémunération, souvent pour des motifs de flexibilité. 


\section{Évaluation du rendement}

L'évaluation du rendement révèle des éléments insoupçonnés dans ces organisations où la réussite des projets détermine directement le niveau de performance des gestionnaires de projets. Comme l'indiquent les résultats du tableau 6 , les responsables des RH de plusieurs entreprises recourent à des outils plutôt sophistiqués d'appréciation et d'évaluation du personnel, ces instruments ayant d'ailleurs été révisés et adaptés au contexte de chaque organisation au cours des cinq dernières années, depuis l'arrivée en poste de près du tiers des DRH interrogés. Cette observation illustre bien les contraintes s'exerçant sur les sociétés de génie-conseil vers une meilleure gestion de leurs $\mathrm{RH}$. On pense ici aux phénomènes de rationalisation et de concentration observés dans ce secteur au cours des 10 dernières années.

\section{TABLEAU 6}

Principaux processus, outils et techniques d'évaluation du rendement $(\mathbf{N}=9)$

\begin{tabular}{|c|c|c|c|c|c|c|}
\hline & & & & 'équenc & & \\
\hline & $0 \%$ & $25 \%$ & $50 \%$ & $75 \%$ & $100 \%$ & Moyenne \\
\hline Méthodes utilisées* & & & & & & \\
\hline Évaluation ouverte (non structurée) & 1 & 0 & 2 & 0 & 6 & 4,1 \\
\hline Échelles de notation & 3 & 0 & 0 & 0 & 6 & 3,7 \\
\hline Direction par objectifs & & & & & & \\
\hline (D.P.O., M.B.O., etc.) & 2 & 1 & 3 & 2 & 1 & 2,9 \\
\hline Liste de comportements & 4 & 0 & 2 & 0 & 3 & 2,8 \\
\hline Incidents critiques & 4 & 1 & 3 & 1 & 0 & 2,1 \\
\hline Critères d'évaluation** & & & & & & \\
\hline Qualité du travail & 1 & 0 & 1 & 1 & 6 & 4,2 \\
\hline Résultats atteints & 1 & 0 & 1 & 2 & 5 & 4,1 \\
\hline Intégrité, éthique professionnelle & 1 & 1 & 0 & 2 & 5 & 4,0 \\
\hline Esprit de collaboration au travail & 1 & 0 & 0 & 5 & 3 & 4,0 \\
\hline Efforts & 1 & 0 & 1 & 4 & 3 & 3,9 \\
\hline Quantité de travail & 1 & 0 & 2 & 4 & 2 & 3,7 \\
\hline Finalités des résultats* & & & & & & \\
\hline $\begin{array}{l}\text { Détermination de la rémunération, } \\
\text { attribution de bonis, autres } \\
\text { récompenses monétaires }\end{array}$ & 0 & 0 & 1 & 2 & 6 & 16 \\
\hline Communication et feedback afin de & & & & & & \\
\hline favoriser le développement des GP & 1 & 0 & 3 & 1 & 4 & 3,8 \\
\hline Détermination des besoins & & & & & & \\
\hline de formation & 1 & 1 & 3 & 0 & 4 & 3,6 \\
\hline Attribution des promotions & 1 & 4 & 0 & 2 & 2 & 3,0 \\
\hline Validation de décisions administratives & 2 & 3 & 2 & 1 & 1 & 2,6 \\
\hline Recours à des mesures disciplinaires & 4 & 1 & 1 & 1 & 2 & 2,6 \\
\hline
\end{tabular}


Le processus se déroule sur une base annuelle [10] ou aux six mois pour les contractuels et à chaque contrat par les clients [3], sans grande procédure et presque toujours par un entretien ouvert, souvent à l'aide d'une grille de notation portant principalement sur la qualité du travail, les résultats atteints, l'intégrité, l'éthique professionnelle ainsi que l'esprit de collaboration au travail et les efforts des gestionnaires de projets. Les autres éléments de contenu touchent, pour la majorité des répondants [8], des critères tels que : points forts et faibles, rentabilité, efficacité, innovation, développement personnel, aptitudes à la gestion, assiduité, ponctualité, compétences, connaissances et habiletés, prise de décision, jugement, respect du budget, développement des affaires et qualités de chef.

Toutes les méthodes formelles ou sophistiquées d'évaluation demeurent sousutilisées : direction par objectifs, liste de comportements ou incidents critiques. Par contre, comme on le constate souvent, cet exercice sert directement à la détermination de la rémunération, à l' attribution de bonis et autres récompenses monétaires et, en second lieu, à maintenir la communication et le feedback afin de favoriser le développement du personnel. En troisième et quatrième positions, on retrouve les objectifs de détermination des besoins de formation et l'attribution des promotions, tandis que la validation de décisions administratives, le recours aux mesures disciplinaires, le réaménagement du travail et la gestion des carrières constituent des finalités marginales de l'évaluation du rendement des gestionnaires de projet.

Par ailleurs, seulement quatre entreprises affirment n'avoir aucune difficulté, ou très peu, liée à l'évaluation du rendement du personnel. Globalement, plusieurs des PME enquêtées [5] tendent à raffiner et à adapter davantage les outils utilisés, à cause de la difficile vérification de l'atteinte des objectifs (par exemple, dans le cadre d'un projet pilote), de la forte résistance face aux discussions ouvertes, des difficultés à appliquer l'autorité et à écouter et, plus rarement, de la difficile formation des évaluateurs (ardue à réaliser et à vérifier). Quatre répondants soulèvent le manque de temps et les reports fréquents du processus d'évaluation, tandis que trois autres firmes se retrouvent avec des évaluations souvent subjectives, informelles, non écrites, liées trop directement aux questions de rémunération. En outre, dans deux cas, la gestion disciplinaire et les congédiements ne s'appuient ni sur des mesures précises, ni sur l'évaluation du rendement. Enfin, les répondants de trois entreprises ne croient ni en son objectif ni en son efficacité, prétendant qu'ils en font chaque jour, mais sans aucun suivi...

\section{Discussion et conclusion}

Les résultats de la présente étude permettent de dresser un premier bilan des pratiques d'acquisition et de conservation des RH prévalant dans des PME en génie-conseil, en plus d'identifier certaines difficultés rencontrées par les personnes responsables de ces pratiques de GRH. L'enquête rejoint aussi certaines observations globales 
contenues dans les rapports produits pour l'Association des ingénieurs-conseils du Québec (1990) et pour l'Association des ingénieurs-conseils du Canada (1994). Notre recherche corrobore aussi plusieurs conclusions des analyses de D'Amboise et Garand (1995) sur les difficultés et besoins prioritaires des PME en GRH. En outre, elle permet de faire émerger de nouvelles pistes à prioriser en fonction des difficultés observées en RH et des besoins propres aux PME de services-conseils. Il convient donc ici d'apporter un éclairage plus grand à certaines tendances lourdes dégagées dans le cadre de l'enquête.

L'interprétation d'une telle gamme de résultats ne s'avère évidemment pas des plus simples. En premier lieu, il parait utile de rappeler les nombreuses contraintes imposées aux firmes de génie-conseil par leur domaine d'activité et leur contexte de gestion de projets. Ainsi, la nature diversifiée, cyclique et imprévisible du secteur (délais, contrats aléatoires, soumissions publiques politisées), ainsi que la persistance de l'insécurité des $\mathrm{RH}$ face aux fins de projets compliquent singulièrement les pratiques de planification des $\mathrm{RH}$, d'analyse des emplois, de recrutement et d'intégration des GP. Liées à l'internationalisation des marchés et à une concurrence accrue (et non traditionnelle) sur les prix, ces contraintes affectent aussi les processus de sélection, de rémunération et d'évaluation du rendement. En effet, les projets deviennent plus complexes et requièrent de plus en plus d'habiletés et de compétences dans des domaines plus proches des ventes et des relations humaines que du génie spécialisé.

En ce qui concerne les pratiques de GRH, on constate que la planification des RH de ces sociétés de génie-conseil paraît un peu plus étoffée que dans la moyenne des PME, bien qu'elle demeure peu formalisée. Ces responsabilités, dévolues au gestionnaire de projets œuvrant sur des projets précis plutôt qu'à un propriétaire-dirigeant de type entrepreneurial, semblent légèrement plus structurées, sans doute à cause de la forme matricielle et de la programmation des projets d'ingénierie. Ainsi, les répondants s'efforcent de mieux planifier et de prévoir l'évolution des marchés afin d'éviter les pénuries de main-d'œuvre spécialisée et de combler à moyen terme leurs éventuels besoins en RH.

L'analyse des emplois correspond sensiblement aux tendances relatées en PME : la moitié des répondants utilisent des descriptions de tâches détaillées. Les DRH interrogés expriment toutefois un certain besoin d'amélioration à ce sujet, notamment en proposant l'élaboration de profils de postes ou de modèles génériques.

Le recrutement des GP semble globalement reposer sur des pratiques conservatrices et peu sophistiquées, l'essentiel du processus étant basé sur le système de relations du dirigeant. Cet état de fait n'exclut toutefois pas un certain nombre de pratiques originales en recrutement, dans ce milieu où tous les DRH se connaissent et disposent d'importantes banques de candidatures. 
En ce qui concerne le processus de sélection, l'échantillon étudié laisse entrevoir des pratiques nettement plus sophistiquées et formalisées que celles retrouvées dans des PME œuvrant dans d'autres secteurs d'activité. Tant pour les entretiens que dans l'analyse des candidatures, les firmes de génie-conseil semblent recourir à des outils de sélection bien davantage élaborés, et occasionnellement contrevérifiés par la suite. Néanmoins, l'implantation de techniques favorisant une meilleure sélection du personnel en fonction des projets demeure une des priorités des DRH de ce secteur.

L'accueil et l'intégration des gestionnaires de projets semblent aussi plus développés, avec plusieurs pratiques originales, l'importance de ce groupe particulier de gestionnaires requérant une attention et une considération accrues. Bien que le taux de roulement des gestionnaires de projets demeure très faible, les répondants signalent un important besoin d'amélioration des mécanismes d'accueil et d'intégration, particulièrement en ce qui a trait aux ingénieures, à la prévalence des contextes de multiprojets et à l'importance croissante des questions liées aux diversités ethniques et culturelles.

Quant aux pratiques de rémunération, on observe en génie-conseil un conservatisme qui semble supérieur à la moyenne des PME, et dans plusieurs cas, une sophistication et une formalisation inférieures. La concentration élevée du secteur (du moins des firmes de 50 à 400 employés), l'individualisation de la rémunération des gestionnaires de projets et l'absence complète de syndicalisation dans ces milieux engendrent des pratiques au cas par cas, induisant certains problèmes de cohérence, d'uniformité et de transparence qui caractérisent moins certaines PME évoluant dans d'autres secteurs d'activité. Malgré cela, les DRH interrogés affirment ne pas percevoir de besoin pressant en cette matière, la satisfaction des clients leur paraissant somme toute élevée et constante. Bien que les avantages sociaux demeurent limités, l'accès aux régimes d'incitation et d'intéressement s'accroît peu à peu et quelques pratiques originales émergent des résultats. Toutefois, les répondants admettent qu'il faudrait accroitre le professionnalisme, la structuration, la diversification et l'intensification de stratégies de rémunération plus compétitives, particulièrement sous forme incitative.

Enfin, le processus d'appréciation et d'évaluation du rendement semble un peu plus développé que celui observé dans des PME d'autres secteurs d'activité, bien qu'il demeure dans bien des cas peu sophistiqué et faiblement formalisé. Quelques exemples de processus étoffés et systématiquement appliqués ont même étonné les chercheurs, les finalités des projets d'ingénierie exigeant des mesures sophistiquées d'évaluation et de suivi, sous les contraintes de délais et de qualité imposées par les clients. Néanmoins, le manque de temps et de sensibilisation des acteurs restreignent substantiellement l'efficacité d'une telle pratique, qui paraît superflue pour certains répondants. 
Globalement, il semble que le retard en GRH semble s'être graduellement résorbé dans les PME de génie-conseil au cours des années 1990. Cette lente évolution vers une meilleure reconnaissance de leur GRH, perceptible et positive, résulte de divers facteurs : les très nombreuses fusions, acquisitions, réorganisations, remises en question corporatives et associatives, le placement des ingénieurs en PME, le passage à l'international ainsi que l'accent mis publiquement sur les RH. Cette tendance s'observe d'ailleurs par l'embauche de nouveaux DRH et l'octroi de «cartes blanches » ambitieuses à leurs meilleurs gestionnaires de ressources humaines. Malheureusement, plusieurs entreprises affichent encore un retard important dans ce domaine : comment pourront-elles conserver leur compétitivité face à des concurrents mieux équipés et plus efficaces grâce à une meilleure gestion de leurs RH? De l'aveu même de certains répondants, la nature conservatrice de l'industrie, tendant à freiner le progrès et l'évolution des processus organisationnels, laisse souvent la GRH un cran derrière les autres priorités de nature technologique ou commerciale. D'une part, les employeurs en génie-conseil souffrent d'une image quelque peu ternie et on peut prévoir à court terme certaines difficultés à combler les besoins futurs en matière de compétences et de connaissances. Cette situation appréhendée s'explique en partie par des lacunes au niveau de la communication, de la planification et de la formation des RH. D'autre part, l'industrie aurait besoin de nouvelles compétences en leadership et en gestion, mais les dirigeants actuels ne semblent pas tous adéquatement préparés à relever les défis inhérents au commerce international. En outre, la rémunération accuse un retard par rapport à celle de certains autres secteurs, tandis que le manque de ressources financières et l'instabilité professionnelle liée aux marchés demeurent des obstacles quasi incontournables.

Par ailleurs, il convient de noter que plus de la moitié des PME enquêtées ont déclaré n'avoir aucune difficulté, ou très peu, en recrutement [7], sélection [8], accueil [9] ou rémunération [8], comparativement à la PRH [3], l'analyse des emplois [5] ou l'évaluation du rendement [4] qui semblent occasionner davantage de difficultés aux entreprises étudiées. Les résultats font ressortir diverses difficultés d'ordre technique, structurel et organisationnel en matière de GRH :

- un manque global d'expertise et la faible sophistication des outils et techniques de GRH ;

- un manque généralisé de temps et de disponibilité chez les gestionnaires de projets et les dirigeants ;

- une faible formalisation des politiques, procédures et pratiques de GRH;

- des réticences chez les dirigeants face à la GRH :

- une relative centralisation autour du propriétaire-dirigeant et des difficultés de délégation; 
- une certaine décentralisation parfois imposée par l'hétérogénéité des projets ;

- une attitude négative et une faible valorisation globale face à la GRH ;

- des difficultés de communication entre les acteurs organisationnels en génie-conseil.

Contrairement aux PME traditionnelles du secteur manufacturier, ces PME de génie-conseil recourent avant toute formalisation à une structuration souvent informelle, inspirée des formes matricielles, qui leur procure la souplesse et la polyvalence nécessaires à la réalisation de projets diversifiés, multiples et simultanés. Ainsi, ces PME de services évoluent-elles jusqu'à une taille de plusieurs dizaines d'employés sans ressentir le besoin de structurer ou de formaliser leur fonction $\mathrm{RH}$, le fonctionnement plus ou moins appliqué en structure matricielle divisant ces RH en petites unités polyvalentes et souvent quasi autonomes. Cette particularité a d'ailleurs posé certaines difficultés aux responsables de $\mathrm{RH}$ rencontrés au cours de l'enquête, ces derniers devant alors verbaliser les pratiques de GRH appliquées au sein des structures de projets par les gestionnaires de projets, hors de leur champ direct d'activité en tant que responsable des RH.

Il faut admettre que cette analyse comporte des limites méthodologiques et conceptuelles dont il convient de préciser la nature. Primo, l'enquête a porté essentiellement sur les pratiques de GRH des gestionnaires de projets en génie-conseil, en tentant le plus possible de faire abstraction des autres catégories de personnel, ce qui n'a pas toujours été facile au cours des entretiens. Secundo, même dans une enquête comme celle-ci, effectuée par entretiens individuels directs, le taux de réponse limite la validité externe de l'étude. Tertio, les caractéristiques des répondants et de leurs réponses imposent des limites à l'interprétation. En effet, l'enquête ne révèle que les perceptions des responsables de $\mathrm{RH}$ de ces $12 \mathrm{PME}$ de génie-conseil. Or, divers résultats de terrain indiquent que les perceptions de ces derniers peuvent ne pas parfaitement coïncider avec celles des clients de la fonction ressources humaines (Tsui, 1987; Tsui et Gomez-Meija, 1988; Tsui et Milkovich, 1987). À cet égard, des résultats obtenus auprès de professionnels œuvrant en contexte de gestion de projets font apparaître d'autres priorités en GRH (Harnois et Fabi, 1994). Il ressort en effet un problème majeur d'insécurité professionnelle face à l'instabilité d'emploi caractérisant les carrières en gestion de projets. L'absence presque totale de planification de carrière semble donc susciter beaucoup d'incertitude et d'insécurité chez les professionnels œuvrant en gestion de projets. De la même façon, ces derniers souhaiteraient massivement participer aux bénéfices de leur entreprise, plutôt que d'être soumis à des pratiques de rémunération plus conservatrices.

Bien entendu, l'ensemble de ces priorités en GRH appelle des actions de gestion qui se heurtent à des contraintes pratiquement incontournables en contexte de gestion de projets, notamment en génie-conseil. On pense ici à la nature cyclique 
de ce secteur d'activité et à l'incapacité de prévoir l'obtention des contrats qui déterminent les besoins en main-d'œuvre (Association des ingénieurs-conseils du Québec, 1990 ; Céré, 1992). On pense aussi aux contraintes financières qui caractérisent plusieurs firmes en génie-conseil, compliquant ainsi le recours ponctuel ou permanent à des spécialistes en GRH.

En conformité avec la théorie de la contingence (Lawrence et Lorsch, 1967), il semble plausible que le faible degré de formalisation de certaines pratiques de GRH et une certaine souplesse d'application soient bien adaptés à ces caractéristiques de l'environnement des entreprises en génie-conseil. Cependant, cette argumentation de contingence pourrait difficilement justifier un excès de conservatisme ou un manque de sensibilité aux problèmes et aux avenues de solutions identifiées par les responsables et par les clients de la fonction $\mathrm{RH}$. Il semble donc indiqué que les dirigeants de ces entreprises, majoritairement de formation en ingénierie, acceptent de modifier certaines de leurs attitudes, d'améliorer leurs connaissances en gestion, notamment en GRH, d'avoir recours à des spécialistes de cette discipline lorsque possible, et d'allouer des ressources suffisantes pour soutenir certaines pratiques de GRH essentielles à l'acquisition et au développement de leur potentiel humain. Sinon, l'expérience des dernières années nous apprend qu'une négligence majeure à cet égard peut, au même titre que des déficiences relatives à la gestion financière ou technologique, menacer la survie même de certaines organisations en génie-conseil. Dans un contexte d'internationalisation de la concurrence et de concentration de la propriété, les organisations gagnantes seront peut-être celles qui auront le mieux géré leur matière grise.

\section{Bibliographie}

AICC-ACEC (1994), « Agir pour prospérer : les ressources humaines dans l'industrie canadienne du génie-conseil », Rapport pour l'Association des ingénieurs-conseils du Canada, novembre, 253 p., et sommaire, 12 p.

AICQ (1990), «Analyse de la situation du génie-conseil québécois et axes d'orientations », Stratem inc., Rapport pour l'Association des ingénieurs-conseils du Québec, juin.

Belcourt, M., A.W. Sherman, G.W. Bohlander et S.A. Snell (1996), Managing Human Resources, Scarborough, Nelson, Canada.

BENOîT, C. et M.D. RousSEAU (1989), «Gestion des ressources humaines dans les PME au Québec », Ministère de la Main-d'œuvre et de la Sécurité du revenu, Direction de la recherche.

BENOÎT, C. et M.D. RouSSEAU (1990), La gestion du personnel dans les PME au Québec - Questionnaire, Gouvernement du Québec, Ministère de la Main-d'œuvre et de la Sécurité du revenu, Direction de la recherche. 
BENOÎT, C. et M.D. RousSEAU (1993), La gestion des ressources humaines dans les PME au Québec : perception des dirigeants, pour le ministère de la Main-d'œuvre, de la Sécurité du revenu et de la Formation professionnelle, Direction de la recherche, Publications du Québec, 315 p.

Byars, L.L. et L.W. RUE (1984), Human Resource and Personnel Management, Homewood, Ill., Irwin.

CAscio, W.F. (1986), Managing Human Resources : Productivity, Quality of Work Life Profits, New York, McGraw-Hill.

CÉRÉ, L. (1992), «L'internationalisation des petites et moyennes firmes de génie-conseil québécoises : un modèle explicatif », Mémoire de maîtrise (M.Sc. Sciences de la gestion), Université de Montréal, École des Hautes Études commerciales, décembre, 183 p.

D'Amboise, G. (1993), «Empirical research on SMEs : the past ten years in Canada », Journal of Small Business \& Entrepreneurship, vol. 10, $\mathrm{n}^{\circ}$ 2, janvier-mars, p. 2-12.

D'AmBoISE, G. et D.J. GARAND (1993), Identification des difficultés et besoins des PME en matière de GRH, Rapport inédit [pour la] Société québécoise de développement de la main-d'œuvre (SQDM), Montréal, octobre, $125 \mathrm{p}$.

D’AMBOISE, G. et D.J. GARAND (1995), « Identification des difficultés et besoins des PME en matière de gestion des ressources humaines », Gestion 2000, vol. 11, n 1, p. 109-132.

Dinsmore, B. (1984), Human Factors in Project Management, New York, American Management Association.

DOLAN, S.L. et R.S. SCHULER (1995), Gestion des ressources humaines au seuil de l'an 2000, Saint-Laurent, Éditions du Renouveau pédagogique.

DOLAN, S.L., V.P. HogUE et J. HARBotTLE (1990), «L'évolution des tendances en gestion des ressources humaines au Québec : étude comparative en fonction des tailles des entreprises », dans R. Blouin (éd.), Vingt-cinq ans de pratique en relations industrielles au Québec, Cowansville, Canada, Éditions Yvon Blais, p. 777-789.

FABI, B. et D.J. GARAND (1994), «L'acquisition des ressources humaines en PME », Revue Internationale P.M.E., vol. 6, $\mathrm{n}^{\text {os }} 3-4$, p. 91-129.

FABI, B. et D.J. GARAND (1997), «La gestion des ressources humaines en PME», dans P.A. Julien et al. (éd.), Les PME : bilan et perspectives, $2^{\mathrm{e}}$ édition, Québec, Presses Inter Universitaires et Paris, Economica, p. 255-303.

FABI, B. et D.J. GARAND (1998), «Human resource management in small business », dans The State of the Art in Small Business and Entrepreneurship (édité par Pierre-André Julien), Aldershot, Ashgate Publishing Limited, p. 330-392.

FABI, B. et R. JACOB (1988), « Perceptions à l'égard des pratiques en gestion des ressources humaines et de l'importance relative des fonctions enseignement, recherche et services à la collectivité : une étude descriptive chez des professeurs d'administration des universités québécoises », Revue Canadienne des Sciences Administratives, vol. 5, $\mathrm{n}^{\mathrm{0}}$ 1, p. 47-56.

FABI, B. et N. PETTERSEN (1992a), «Human resource management practices in project management », International Journal of Project Management, vol. 10, $\mathrm{n}^{\mathrm{o}} 2$, mai, p. $81-88$. 
FABI, B. et N. PETTERSEN (1992b), «Le diagnostic de la fonction gestion des ressources humaines », Actes du Congrès, VII ${ }^{\mathrm{e}}$ Congrès international de l'Association de psychologie du travail de langue française (APTLF), Strasbourg, p. 262-270.

FABI, B. et N. PETTERSEN (1994), «Diagnostic de la fonction gestion des ressources humaines en contexte de gestion de projets, questionnaire», Document de travail, Trois-Rivières, UQTR, Département d'administration et d'économique, mai, $42 \mathrm{p}$.

FABI, B., D.J. GARAND et N. PETTERSEN (1993), « La gestion des ressources humaines en PME : proposition d'un modèle de contingence », dans M. Amami et al. (éd.), Compétitivité des $P M E$ et marchés sans frontière, $1^{\text {er }}$ Congrès international francophone de la PME, Carthage, Tunisie, p. 216-229.

FILION, L.J. (1990), « Recherche, épistémologie et méthodologies de recherche en sciences administratives : dix préceptes pour guider nos réflexions face aux années 90 », Revue PMO, vol. 5, $\mathrm{n}^{\circ}$ 2, septembre, p. 46-54.

GARAND, D.J. (1993), Les pratiques de gestion des ressources humaines (GRH) en petites et moyennes entreprises (PME) : une synthèse conceptuelle et empirique, Rapport de recherche, Trois-Rivières, UQTR, Département d'administration et d'économique, Groupe de recherche en économie et gestion des PME (GREPME), mars, 386 p.

GARAND, D.J. et B. FABI (1992), «État de la recherche. Les pratiques de gestion des ressources humaines (GRH) en PME », Revue Organisation, vol. 2, $\mathrm{n}^{\circ}$ 1, automne, p. 61-99.

GARAND, D.J. et B. FABI (1994), «La conservation des ressources humaines en PME», Revue Internationale P.M.E., vol. 7, $\mathrm{n}^{\circ}$ 1, p. 85-126.

HARNOIS, É. (1993), «Attentes des professionnels œuvrant dans un contexte de gestion de projet à l'égard de la gestion des ressources humaines », Travail dirigé (M.Sc.) maîtrise en gestion de projet, Université du Québec à Trois-Rivières, 128 p.

HARNOIS, É. et B. FABI (1994), «Les attentes des professionnels œuvrant en contexte de gestion de projet à l'égard de la gestion des ressources humaines », Cahier de recherche $\mathrm{n}^{\circ}$ GEP-1994-03, Trois-Rivières, UQTR, Département d'administration et d'économique, Équipe de recherche en gestion de projets (ERGEP), avril, $26 \mathrm{p}$.

HORNSBY, J.S. et D.F. KURATKO (1990), « Human resource management in small business : critical issues for the 1990's ", Journal of Small Business Management, vol. 28, n 3, p. 9-18.

JACKSON, S.E., R.S. SCHULER et J.C. RIVERO (1989), «Organizational characteristics as predictors of personnel practices », Personnel Psychology, vol. 42, no 4, p. 727-786.

JACOB, R. et J. DUCHARME (éd.) (1995), Changement technologique et gestion des ressources humaines. Fondements et pratiques, Montréal, Gaëtan Morin Éditeur, 344 p.

LAWRENCE, D.B. (1979), « Personnel ratios : 1978 food-for-thought figures » Personnel, janvier-février, p. 4-10.

LAWRENCE, P.R. et J.W. LORSCH (1967), Organization and Environment, Homewood, Ill., Irwin.

MCEvoy, G.M. (1984), « Small business personnel practices », Journal of Small Business Management, vol. 22, $\mathrm{n}^{\circ} 4$, p. 1-8. 
MEALIEA, L.W. et D. LEE (1990), « Contemporary personnel practices in Canadian firms », Relations Industrielles, vol. 35, $\mathrm{n}^{\mathrm{O}} 3$, p. 410-420.

Milkovich, G.T. et W.F. Glueck (1985), Personnel/Human Resource Management : A Diagnostic Approach, Plano, Texas, Business Publications Inc.

MurRaY, V.V. et D.E. DIMICK, (1978), « Contextual influences on personnel policies and programs : an explanatory model », Academy of Management Review, vol. 3, $\mathrm{n}^{\circ} 4$, p. 750-761.

PERETTI, J.M. (1994), Ressources humaines, Paris, Vuibert.

Petit, A., L. Bélanger, C. Bénadou, I.L. Bergeron et R. FAuCher (1989), Gestion stratégique et opérationnelle des ressources humaines, Montréal, Gaëtan Morin Éditeur.

PMI Standards Committee (1996), A Guide to the Project Management Body of Knowledge, Upper Darby, PA, Project Management Institute.

RouX, B. (1994), «L'évaluation du rendement en contexte de gestion de projet : élaboration d'un instrument d'évaluation continue », Rapport de recherche, maîtrise en gestion de projet (M.Sc.), Université du Québec à Trois-Rivières, 115 p.

SAHA, S.K. (1989), «Variations in the practice of human ressource management : a review », Revue Canadienne des Sciences Administratives, vol. 6, $\mathrm{n}^{\circ}$ 3, p. 37-45.

Sekiou, L., L. Blondin, B. FABI, F. Chevalier et C.H. Besseyre Des Horts (1992), Gestion des ressources humaines, Montréal, Les Éditions $4 \mathrm{~L}$ inc.

ThibodeAU, P. (1996), «La gestion des ressources humaines en contexte de gestion de projet dans des firmes de services-conseils en technologie de l'information de la région de Québec : un diagnostic », Travail dirigé, maîtrise en gestion de projet (M.Sc.), Université du Québec à Trois-Rivières, juillet, 133 p.

TSUI, A.S. (1984), « Personnel department effectiveness : a tripartite approach », Industrial Relations, vol. 23, $\mathrm{n}^{\circ}$ 2, p. 184-197.

TSUI, A.S. (1987), « Defining the activities and effectiveness of the human resource department : a multiple constituency approach », Human Resource Management, vol. 26, $\mathrm{n}^{\circ} 1$, p. 35-69.

Tsui, A. et L. GoMEZ-MEIJA (1988), «Evaluating human resource effectiveness », dans L. Dyer (éd.), Human Resource Management : Evolving Roles and Responsibilities, Washington, Bureau of National Affairs, p. 187-227.

Tsui, A. et G. MILKOVICH (1987), «Personnel department activities : constituency perspectives and preferences », Personnel Psychology, vol. 40, $\mathrm{n}^{\circ}$ 3, p. 519-537.

Tyson, S. et A. FELL (1986), Evaluating the Personnel Function, Londres, Hutchinson.

ULRICH, D. (1989), «Assessing human resource effectiveness : stakeholder, utility and relationship approaches », Human Resource Planning, vol. 12, p. 301-315. 